\title{
Dwarf spheroidals in the M 81 group - Metallicity distribution functions and population gradients
}

\author{
S. Lianou ${ }^{1, \star}$, E. K. Grebel ${ }^{1}$, and A. Koch ${ }^{2}$ \\ 1 Astronomisches Rechen-Institut, Zentrum für Astronomie der Universität Heidelberg, Mönchhofstrasse 12-14, \\ 69120 Heidelberg, Germany \\ e-mail: [lianou;grebel]@ari.uni.heidelberg.de \\ 2 University of Leicester, Department of Physics and Astronomy, University Road, LE1 7RH Leicester, UK \\ e-mail: ak326@astro.le.ac.uk
}

Received 28 September 2009 / Accepted 25 February 2010

\section{ABSTRACT}

\begin{abstract}
Aims. We study the dwarf spheroidal galaxies in the nearby M 81 group in order to construct their photometric metallicity distributions and to investigate the potential presence of population gradients. We select all the dwarf spheroidals with available Hubble Space Telescope / Advanced Camera for Surveys archival observations, nine in total.

Methods. We interpolate isochrones so as to assign a photometric metallicity to each star within a selection box in the color-magnitude diagram of each dwarf galaxy. We assume that the dwarf spheroidals contain mainly an old stellar population. In order to search for metallicity gradients, we examine the spatial distribution of two stellar populations that we separate according to their metallicities. Results. As a result, we present the photometric metallicity distribution functions, the cumulative histograms and smoothed density maps of the metal-poor and metal-rich stars as well as of the intermediate-age stars.

Conclusions. From our photometric data we find that all the dwarf spheroidals show a wide range in metallicities, with mean values that are typical for old and metal-poor systems, with the exception of one dwarf spheroidal, namely IKN. Some of our dwarf spheroidals exhibit characteristics of transition-type dwarfs. Compared to the Local Group transition type dwarfs, the M 81 group ones appear to have mean metallicity values slightly more metal-rich at a given luminosity. All the dwarf spheroidals considered here appear to exhibit either population gradients or spatial variations in the centroids of their metal-poor and metal-rich population. In addition, there are luminous AGB stars detected in all of them with spatial distributions suggesting that they are well mixed with the old stars.
\end{abstract}

Key words. galaxies: dwarf - galaxies: stellar content - galaxies: structure - galaxies: interactions - galaxies: groups: individual: M81 group

\section{Introduction}

The dwarf galaxies within our Local Group (LG) have been the subject of intensive spectroscopic and photometric observations in different wavelength regimes and thus are well studied objects. Their study has been facilitated by the proximity of these dwarfs so that individual stars may even be resolved down to the main sequence turn-off, depending on their distance. Thus, extending the studies to dwarf galaxies in nearby groups with different environment and comparing their properties are of great importance in order to understand the main drivers of their evolution. In addition, the derived properties can provide a way to constrain models of galaxy formation as well as chemical evolutionary models

In this respect, the M 81 group is an interesting target: despite several differences, it bears close resemblance to our LG. The similarity of the M 81 group to our LG lies in its binary structure (Karachentsev et al. 2002), while its difference is mainly due to the recent interactions between its dominant consituents as revealed by the formation of tidal tails and bridges detected in HI observations (Appleton et al. 1981; Yun et al. 1994). With

* Fellow of the Heidelberg Graduate School of Fundamental Physics (HGSFP) and member of the International Max Planck Research School (IMPRS) for Astronomy \& Cosmic Physics at the University of Heidelberg. a mean distance of $\sim 3.7 \mathrm{Mpc}$ (Karachentsev et al. 2002), the M 81 group is one of the nearest groups to our own LG. It consists of about 40 dwarfs of both early-type and late-type, with the addition of 12 recently discovered dwarf candidates (Chiboucas et al. 2009).

Here we focus on the dwarf spheroidal galaxies (dSphs) in the M81 group with available Hubble Space Telescope (HST)/Advanced Camera for Surveys (ACS) archival data. The dSphs are objects with low surface brightness and poor in gas content. For a summary of their properties we refer to Grebel et al. (2003; and references therein). We use their colormagnitude diagrams (CMDs) to derive the photometric metallicity distribution functions (MDFs) and search for the potential presence of population gradients in the M 81 group dSphs.

The use of the CMD to infer the star formation histories and MDFs is a very powerful tool. For nearby groups at distances, where individual red giants are beyond the reach of spectroscopy even with 8-10 m class telescopes, CMDs are the best means to derive evolutionary histories. With the use of HST observations of adequate depth, the upper part of the red giant branch (RGB) can be resolved into single stars. Many studies have derived the photometric MDFs of distant LG dSphs (for example Cetus by Sarajedini et al. 2002; And VI and And VII by Grebel \& Guhathakurta 1999) from their CMDs. A similar work 
to derive the photometric MDFs for dwarf galaxies in nearby groups has not been done so far.

The search for radial population gradients in LG dwarf galaxies has been favoured by the fact that the resolved stellar populations reach the horizontal branch or extend even below the main-sequence turn-off depending on the distance of the dwarf, permitting one to use a variety of different stellar tracers. There are several studies for population gradients in the LG dwarfs and as an example of such studies we refer to the work done by Hurley-Keller et al. (1999), Harbeck et al. (2001), Battaglia et al. (2006) (photometric) and Tolstoy et al. (2004), Koch et al. (2006) (spectroscopic). There is not any study so far searching for population gradients in nearby group dwarf galaxies.

This paper is structured as follows. In Sect. 2 we present the observations, in Sect. 3 we show our results, in Sect. 4 we discuss our main findings and in Sect. 5 we present our conclusions.

\section{Observations}

We use HST/ACS archival data that were retrieved through the Multimission Archive at STScI (MAST). The details of the datasets used are listed in Table 1, where the columns show: (1) the galaxy name, (2) and (3) equatorial coordinates of the field centers (J2000.0), (4) the number of the Program ID and the PI, (5) the ACS / WFC filters used, and (6) the total exposure time for each filter.

The data reduction was carried out using Dolphot, a modified version of the HSTphot photometry package (Dolphin 2000) developed specifically for ACS point source photometry. The reduction steps followed are the ones described in the ACS module of the Dolphot manual. In the Dolphot output photometric catalogue, only objects with $S / N>5$ and "type" equal to 1 , which means "good stars", were allowed to enter the final photometric catalogue. The "type" is a Dolphot parameter that is used to distinguish objects that are classified as "good stars", "elongated objects", "too sharp objects" and so on. After this first selection, quality cuts were applied so as to further clean the photometric catalogue. These cuts were based on the distributions of the sharpness and crowding parameters, as suggested in the Dolphot manual and also in Williams et al. (2009). Guided by these distributions, we use for the sharpness parameter the restriction of $\mid$ sharpness $_{\text {filter }}+$ sharpness $_{F 814 W} \mid<\alpha$, where $(1.0<\alpha<1.5)$ depending on the $\mathrm{dSph}$, and for the crowding parameter the requirement $\left(\right.$ Crowding filter + Crowding $\left._{F 814 W}\right)<1.0$, where "filter" corresponds to either the $F 606 W$ or the $F 475 W$ filter. These selections were made so as to ensure that only stellar objects have entered our final photometric catalogue. The number of stars recovered after applying all the photometric selection criteria are listed in Table 2, Col. (3).

The photometry obtained with Dolphot provides magnitudes in both the ACS / WFC and the Landolt UBVRI photometric systems using the transformations provided by Sirianni et al. (2005) for the $U B V R I$ system. In the analysis presented throughout this work, we chose to use the ACS / WFC filter system. Therefore, if we use data from the literature computed in the UBVRI photometric system, we transform them to the ACS/WFC system. There are two cases where this is necessary. The first case is the extinction. The galactic foreground extinction in the $V$ band and $I$-band, $A_{I}$ and $A_{V}$, taken from Schlegel et al. (1998) through NED, are transformed into the ACS / WFC system. For the transformation, we use the corresponding extinction ratios $A(P) / E(B-V)$ for a $\mathrm{G} 2$ star, where $A(P)$ corresponds to the extinctions in the filters $F 814 W$ and $F 606 W$ (or $F 475 W$ ), which are provided by Sirianni et al. (2005; their Table 14). We note that the assumption of a largely color-independent reddening for the RGB is justified since theoretical models indicate that the expected effect of the change of color accross the RGB amounts to at most 0.01 in $E(V-I)$ for our data (see Grebel \& Roberts 1995). We multiply these extinction ratios with the $E(B-V)$, in order to finally get the extinctions in the ACS filters. The transformed values, $A_{F 814 W}$ and $A_{F 606 W}$ (or $A_{F 475 W}$ ), are listed in Table 2, Cols. (6) and (7) respectively.

The second case is the I-band tip of the RGB (TRGB), which we transform to the $F 814 W$-band TRGB in the following way. As already mentioned, Dolphot provides the magnitudes both in the instrumental ACS/WFC system and in the transformed $U B V R I$. Thus, in the range of magnitudes near the $I$ band TRGB, we compute the difference in magnitudes between the $I$-band and $F 814 W$-band. This difference is 0.01 mag for all the dSphs except for DDO 44 and DDO 78, where the difference is $-0.015 \mathrm{mag}$. The $F 814 W$-band TRGB is then equal to the sum of this difference and the $I$-band TRGB. We confirm further more this approach of estimating the $F 814 \mathrm{~W}$-band TRGB by applying a Sobel-filtering technique to the luminosity function of some of the dSphs (Lee et al. 1993; Sakai et al. 1996) estimating the location of the $F 814 W$-band TRGB. We find that these approaches give values that are in good agreement, with a mean difference between them of the order of $0.05 \mathrm{mag}$.

We list the global properties of the present dSph sample in Table 2. The columns contain the following information: (1) the galaxy name, (2) the galaxy type, (3) the number of stars detected after applying all the photometric selection criteria, (4) the visual absolute magnitude $M_{V}$ of each galaxy adopted from Karachentsev et al. (2000, 2001), Alonso-Garcia et al. (2006), Georgiev et al. (2009), (5) the I-band TRGB adopted from Karachentsev et al. (2006, 2001, 2000, 1999), (6) and (7) the foreground extinction derived by us for the ACS/WFC filters $F 814 W, F 606 W$ and $F 475 W$, as described in Sect. 2, (8) the true distance moduli adopted from Karachentsev et al. (2006, 2001, 2000, 1999), (9) the deprojected distance of the dSphs from the M 81 galaxy, $R$, adopted from Karachentsev et al. (2002), (10) the effective radius, $r_{\text {eff }}$ adopted from Sharina et al. (2008) and Karachentseva et al. (1987). The dSphs in Table 2 are sorted according to their $M_{V}$ value.

Finally, the pixel scale of the ACS/WFC is $0.05^{\prime \prime}$ with a field of view of $202^{\prime \prime} \times 202^{\prime \prime}$ or $4096 \times 4096$ pixels. Thus for the mean distance of $\sim 3.7 \mathrm{Mpc}$ of the M 81 group (Karachentsev et al. 2002) this field of view corresponds to $3.6 \times 3.6 \mathrm{kpc}$, or simply 1 pixel corresponds to roughly $1 \mathrm{pc}$.

\section{Results}

\subsection{Color-magnitude diagrams}

We show the CMDs of the nine dSphs in Fig. 1, where we note the difference in the $x$-axis. The proximity of the M81 group and the depth of the observations allow us to resolve the upper part of the RGB into individual stars. The most prominent feature seen in our CMDs is the RGB. We note the presence of stars above the TRGB, which is indicated in Fig. 1 with a dashed line. These stars are most likely luminous AGB stars, which indicate the presence of stellar populations in an age range from 1 Gyr up to less than 10 Gyr. In addition, some of the dSphs appear to have bluer stars that probably belong to a younger main sequence. The dwarfs in our sample are classified as dSphs (Karachentsev et al. 2004), with the exception of KDG 61, KDG 64, DDO 71, and HS 117 which are classified as 

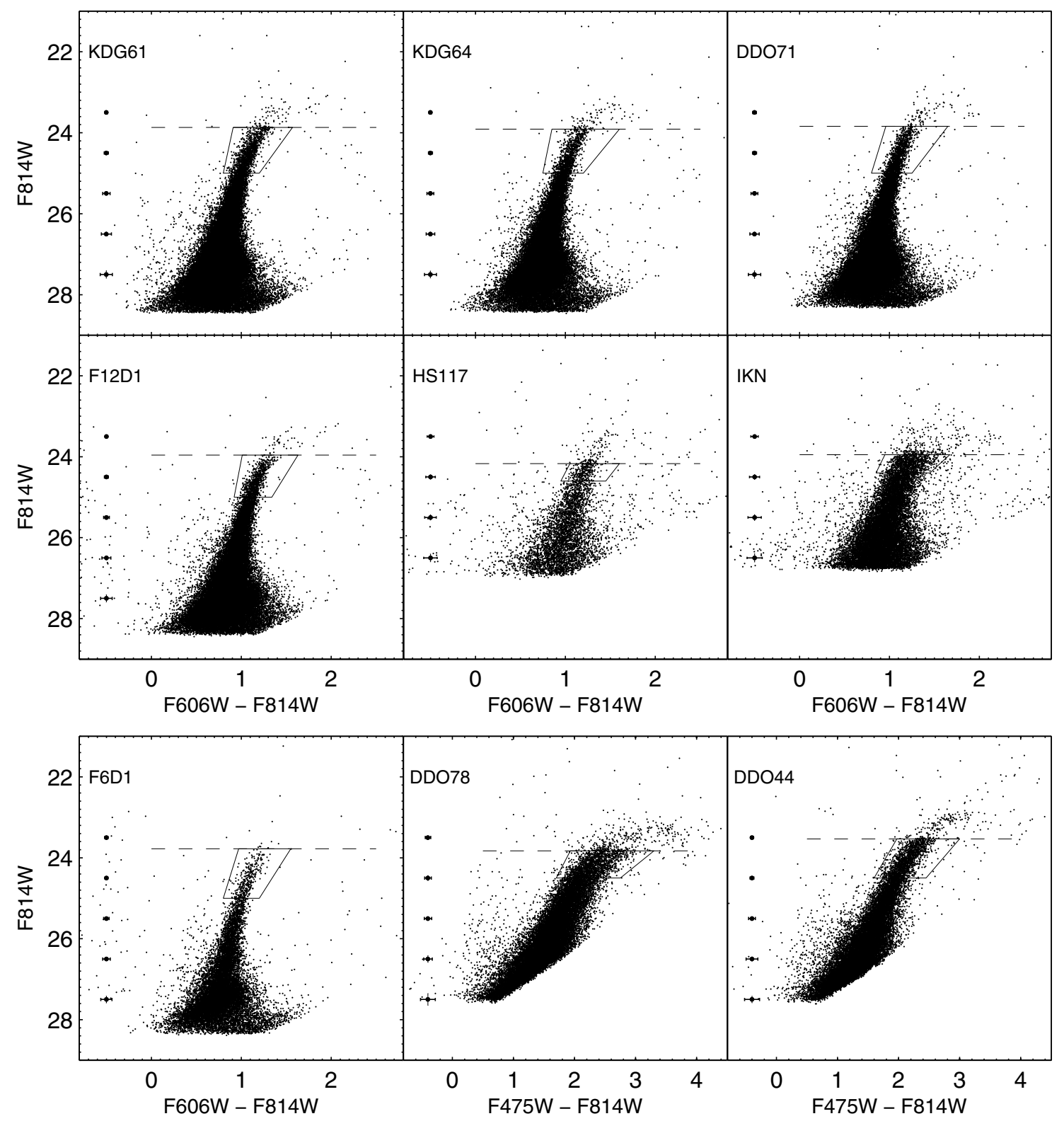

Fig. 1. Color-magnitude diagrams for the nine dSphs. The horizontal dashed lines show the location of the TRGB. The crosses on the left hand side correspond to the photometric errors as derived from artificial star tests. The boxes enclose the stars for which we derive the photometric metallicities.

transition-types (dIrr/dSph) as they have detectable HI content (Huchtmeier \& Skillman 1998; Boyce et al. 2001) or H $\alpha$ emission (Karachentsev \& Kaisin 2007; Karachentsev et al. 2006).

\subsection{Photometric metallicity distribution functions}

We show the photometric MDFs for the nine dSphs in Fig. 2. These are constructed using linear interpolation between Dartmouth isochrones (Dotter et al. 2008) with a fixed age of 12.5 Gyr. We use Dartmouth isochrones, since they give the best simultaneous fit to the full stellar distribution within a CMD as demonstrated by e.g. Glatt et al. (2008a, 2008b). We chose the fixed age of 12.5 Gyr since the RGB in these dSphs may be assumed to consist of mainly old stars in an age range of about 10 Gyr to 13 Gyr. The assumption of an old isochrone is also justified by the omnipresence of old stellar populations in all of the LG dSphs (Grebel 2001; Grebel \& Gallagher 2004) and by the comparatively small number of luminous AGB stars above the TRGB.

The choice of $12.5 \mathrm{Gyr}$ is an assumption we are making in order to estimate the MDFs for each dwarf, while the choice of another age in the above range would not considerably affect our results. Indeed, the colors of the stars on the RGB are mostly affected by metallicity differences rather than age differences (see for example Caldwell et al. 1998; Harris et al. 1999 (their Fig. 6); Frayn \& Gilmore 2002 (their Fig. 2)). Thus the observed spread in the RGB color is likely caused by a metallicity spread rather than an age spread, justifying our choice of an constant age isochrone.

The isochrone metallicities we used range from -2.50 dex to $-0.50 \mathrm{dex}$ with a step of $0.05 \mathrm{dex}$. The isochrone step we used is chosen such that it is smaller than the photometric errors. Representative photometric error bars are indicated with crosses in Fig. 1. To account for the influence of crowding and the photometric quality, we conducted artificial star tests. For 

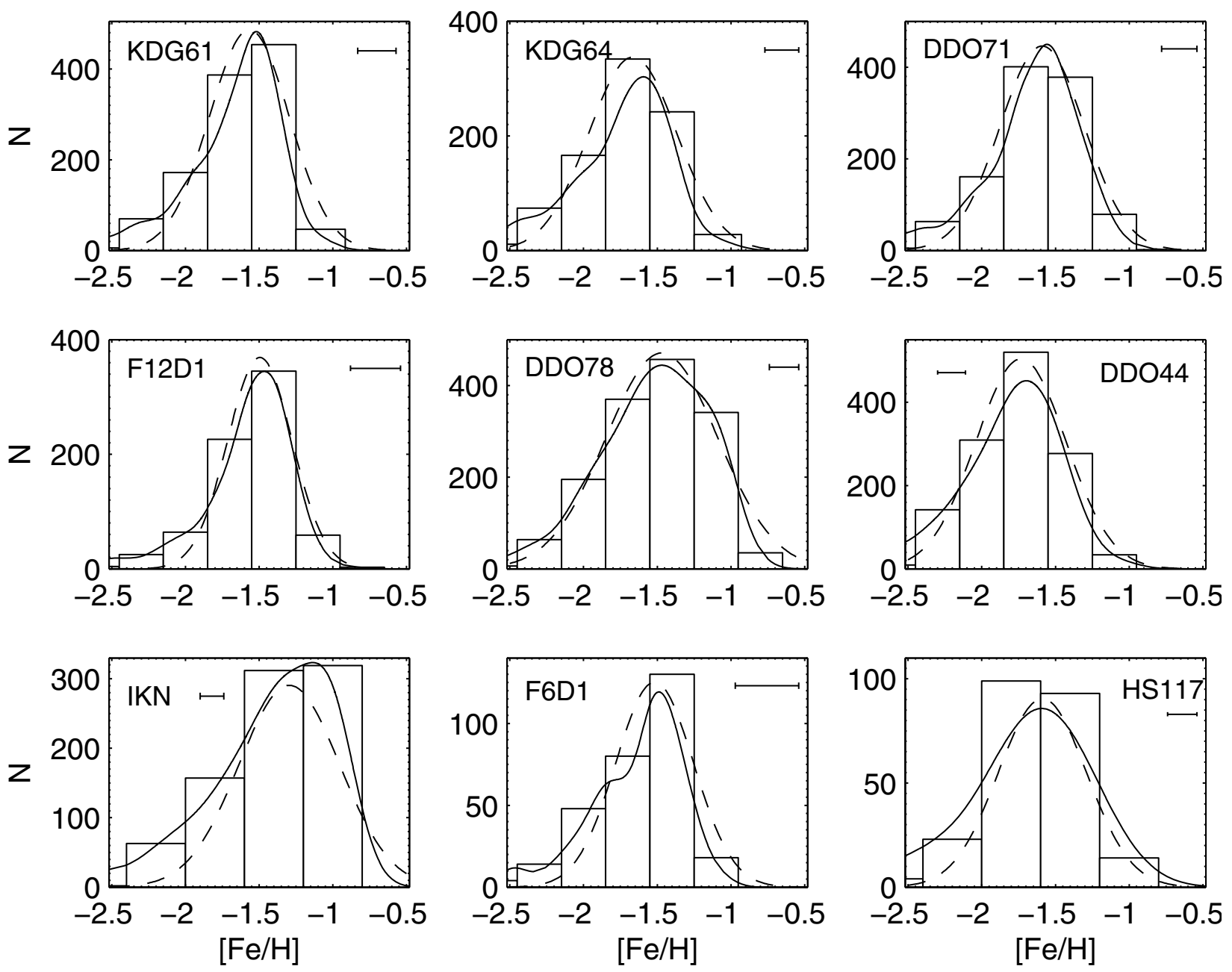

Fig. 2. Metallicity distribution functions for the nine dSphs sorted by their absolute $M_{V}$ magnitude, from top to bottom and from left to right. The solid lines show the metallicity distribution convolved with the errors in metallicity. The dashed lines show the fitted Gaussian distributions. The error bars in the upper right corner, or upper left in the case of DDO 44, show the $1 \sigma$ spread for the weighted mean metallicity we derive from our data. Note the different scaling of the individual $y$-axes.

that purpose, we used the utilities provided and as described in Dolphot.

Given the lack of any spectroscopic information, this method of deriving the MDFs is in general fairly accurate as discussed in Frayn \& Gilmore (2002). In practice, we interpolate between the two closest isochrones bracketing the color of a star, in order to find the metallicity of that star.

We only select stars within a box plausibly containing stars on the upper RGB to construct the galaxies' MDF. The bright magnitude limit of the box is chosen to exclude the stars brighter than the TRGB which belong mainly to the luminous AGB phase. The faint magnitude limit of the box is chosen to fulfil the requirement that the formal error in the derived $[\mathrm{Fe} / \mathrm{H}]$ is less than 0.15 dex, or 0.2 dex in the case of IKN and HS 117 when the photometric errors are taken into account. We employ a different selection criterion in the case of IKN and HS 117 in order to have a significant number of stars in their sample as compared to the one of the remaining $\mathrm{dSphs}$. The selection criterion based on the metallicity formal error depends on the depth of the observations. In our data sample we distinguish three categories, from now on referred to as depth categories. The first depth category contains KDG 61, KDG 64, DDO 71, F6D1 and F12D1. The second depth category contains DDO 44 and DDO 78. The third depth category contains IKN and HS 117. Each depth category contains those dSphs that belong to the same Program ID and thus have the same filters and roughly the same exposure times, as listed in Table 1.

In order to estimate the faint magnitude limit for each dSph's RGB box as a function of the error in $[\mathrm{Fe} / \mathrm{H}]$, we proceed as follows. We extend the faint limit of the bounding box to a magnitude limit of 26 in $F 814 \mathrm{~W}$ for all the dSphs. We compute the $[\mathrm{Fe} / \mathrm{H}]$ for all the stars within each dSph's box, as well as the corresponding errors in metallicity. We show the derived mean errors in metallicity versus the $F 814 W$-band magnitude in Fig. 3 for KDG 61, DDO 44, IKN and HS 117, which are chosen here as representative examples of the three depth categories. In the case of IKN and HS 117, which belong to the third depth category, we show the corresponding plots for both since the requirement of 0.20 dex leads to slightly different faint limits of the RGB box. Based on these plots and on the metallicity requirements, we choose 25 and $24.5 \mathrm{mag}$ as faint limit for the first and second depth category, while in the case of IKN and HS 117 we choose 24.4 and $24.6 \mathrm{mag}$, respectively. The choice of these limits corresponds to an error in color of less than 0.02 mag for the first depth category and less than 0.07 mag for the remaining two depth categories. We note that the difference in the error in 
Table 1. Log of observations.

\begin{tabular}{lccccc}
\hline \hline Galaxy & RA (J2 000.0) & Dec (J2000.0) & Program ID / PI & ACS / WFC filters & $\begin{array}{c}\text { Exposure time } \\
(\mathrm{s})\end{array}$ \\
$(1)$ & $(2)$ & $(3)$ & $(4)$ & $(5)$ & $(6)$ \\
\hline KDG 61 & 095703.10 & +683531.0 & GO 9884 / Armandroff & $F 606 W / F 814 W$ & $8600 / 9000$ \\
KDG 64 & 100701.90 & +674939.0 & $\ldots$ & $\ldots$ & $\ldots$ \\
DDO 71 & 100506.40 & +663332.0 & $\ldots$ & $\ldots$ & $\ldots$ \\
F12D1 & 095010.50 & +673024.0 & $\ldots$ & $\ldots$ & $\ldots$ \\
F6D1 & 094510.00 & +684554.0 & $\ldots$ & $F 606 W / F 814 W$ & $1200 / 900$ \\
HS 117 & 102125.20 & +710651.0 & SNAP 9771 / Karachentsev & $\ldots$ \\
IKN & 100805.90 & +682357.0 & $\ldots$ & $\ldots$ & $\ldots$ \\
DDO 78 & 102628.00 & +673935.0 & GO 10915/Dalcanton & $F 475 W / F 814 W$ & $2274 / 2292$ \\
DDO 44 & 073411.50 & +665247.0 & $\ldots$ & $\ldots$ & $2361 / 2430$ \\
\hline
\end{tabular}

Notes. Units of right ascension are hours, minutes, and seconds, and units of declination are degrees, arcminutes and arcseconds.

Table 2. Global Properties (see text for references).

\begin{tabular}{lccccccccc}
\hline \hline Galaxy & Type & $N_{*}$ & $\begin{array}{c}M_{V} \\
(\mathrm{mag})\end{array}$ & $\begin{array}{c}I_{\mathrm{TRGB}} \\
(\mathrm{mag})\end{array}$ & $\begin{array}{c}A_{F 814 W} \\
(\mathrm{mag})\end{array}$ & $\begin{array}{c}A_{F 606 W}{ }^{a} \\
(\mathrm{mag})\end{array}$ & $\begin{array}{c}(m-M)_{\mathrm{O}} \\
(\mathrm{mag})\end{array}$ & $\begin{array}{c}R \\
(\mathrm{kpc})\end{array}$ & $\begin{array}{c}r_{\mathrm{eff}} \\
(\prime \prime)\end{array}$ \\
$(1)$ & $(2)$ & $(3)$ & $(4)$ & $(5)$ & $(6)$ & $(7)$ & $(8)$ & $(9)$ & $(10)$ \\
\hline KDG 61 & dIrr / dSph & 53543 & -13.87 & $23.86 \pm 0.15$ & 0.131 & 0.202 & $27.78 \pm 0.15$ & 44 & 48 \\
KDG 64 & dIrr / dSph & 38012 & -13.43 & $23.90 \pm 0.15$ & 0.099 & 0.152 & $27.84 \pm 0.15$ & 126 & 28 \\
DDO 71 & dIrr / dSph & 37291 & -13.22 & $23.83 \pm 0.15$ & 0.173 & 0.267 & $27.72 \pm 0.15$ & 211 & 59 \\
F12D1 & dSph & 39519 & -12.84 & $23.95 \pm 0.15$ & 0.263 & 0.404 & $27.71 \pm 0.15$ & 181 & 31 \\
DDO 78 & dSph & 21073 & -12.83 & $23.85 \pm 0.15$ & 0.040 & 0.079 & $27.85 \pm 0.15$ & 223 & 38 \\
DDO 44 & dSph & 19357 & -12.56 & $23.55 \pm 0.15$ & 0.075 & 0.149 & $27.52 \pm 0.15$ & 901 & 28 \\
IKN & dSph & 14600 & -11.51 & $23.94 \pm 0.15$ & 0.111 & 0.171 & $27.87 \pm 0.18$ & 110 & $\ldots$ \\
F6D1 & dSph & 14260 & -11.46 & $23.77 \pm 0.14$ & 0.144 & 0.222 & $27.66 \pm 0.17$ & 218 & 32 \\
HS 117 & dIrr / dSph & 4596 & -11.31 & $24.16 \pm 0.15$ & 0.210 & 0.323 & $27.99 \pm 0.18$ & 204 & 29 \\
\hline
\end{tabular}

Notes. ${ }^{(a)}$ Or $A_{F 475 W}$ in the case of DDO 44 and DDO 78.
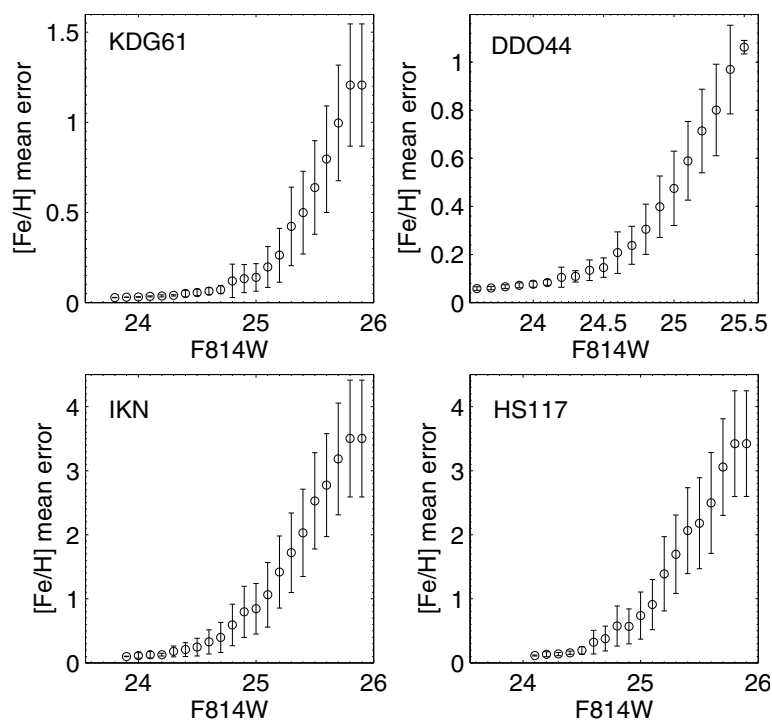

Fig. 3. Mean error in metallicity versus the $F 814 W$-band magnitude. The circles indicate the mean error in metallicity in each magnitude bin, while the error bars indicate the standard deviation of the errors.

color is due to the different exposure times for each dSph data set, which are listed in the Col. (6) of Table 1. The RGB boxes used in each dSph are drawn in Fig. 1.

In Fig. 4, left panel, we plot the RGB box used in the case of the dwarf KDG 64 as well as a subset of the isochrones used for the interpolation method, here ranging from $-2.50 \mathrm{dex}$ to $-0.80 \mathrm{dex}$. The step remains $0.05 \mathrm{dex}$. The grid of the
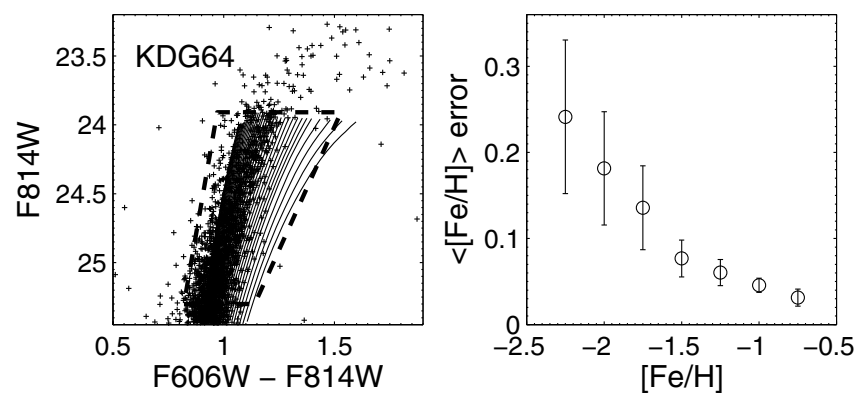

Fig. 4. Left panel: color-magnitude diagram for KDG 64 zoomed in the RGB part to show the stars selected in the box, shown with the dashed line, for which we compute their photometric metallicities. In the same figure we overplot with solid lines a subset of the isochrones used for the interpolation method, with metallicities ranging from -2.50 dex to -0.80 dex. Right panel: the error in metallicity versus the $[\mathrm{Fe} / \mathrm{H}]$ as derived with the use of Monte Carlo simulations. The circles indicate the mean metallicity in each metallicity bin, while the error bars indicate the standard deviation of the mean.

theoretical isochrones we use is fine enough that the spacing between them is kept nearly vertical. We correct the magnitudes and colors of the theoretical isochrones for foreground Galactic extinction and for the distance modulus of each $\mathrm{dSph}$. The $A_{F 814 W}$ and $A_{F 606 W}$ (or $A_{F 475 W}$ in the case of DDO 44 and DDO 78) that we calculate and the true distance moduli are listed in Table 2, Cols. (6) and (7) for the extinction and (8) for the distance moduli. The $I$-band TRGB values shown in Col. (5) of the same table were used to compute the $F 814 W$-band TRGB values, as explained already in Sect. 2. 
We note in Fig. 4 the presence of stars within the RGB box bluewards of the most metal-poor isochrone available from the Dartmouth set of isochrones. These stars are not used to construct the MDF. The existence of such stars may indicate the presence of more metal-poor RGB stars or of old AGB stars, with ages typically greater or equal to 10 Gyr. Such old AGB stars that have the same luminosity as RGB stars were also noted, for example, by Harris et al. (1999) while constructing the MDF for stars in a halo field of the giant elliptical NGC 5128. It is expected that at most $22 \%$ of the stars in the RGB selection box, and within 1 mag below the TRGB, are actually old AGB stars (Durrell et al. 2001; Martinez-Delgado \& Aparicio 1997; and references therein). In order to quantify the effect of the presence of such stars, we construct the MDF of KDG 61 using Padova isochrones (Girardi et al. 2008; Marigo et al. 2008), which also include the AGB phase. We run the interpolation code once using isochrones that only include the RGB phase and once with isochrones that only include the AGB phase, for a constant age of $12.5 \mathrm{Gyr}$ and a range in metallicities from $[\mathrm{Fe} / \mathrm{H}]=-2.36 \mathrm{dex}$ (or $Z=0.0001$ ) to $[\mathrm{Fe} / \mathrm{H}]=-0.54 \mathrm{dex}$ (or $Z=0.006$ ), with a step of $0.1 \mathrm{dex}$ in $[\mathrm{Fe} / \mathrm{H}]$. The derived mean values differ only by 0.04 dex in $[\mathrm{Fe} / \mathrm{H}]$ with a mean of $\langle[\mathrm{Fe} / \mathrm{H}]\rangle=-1.24$ dex for the MDF constructed using isochrones that include only the RGB phase. The MDF of the stars that were fit using isochrones that include only the AGB phase becomes more metal-rich. Furthermore, the derived $1 \sigma$ spreads in $[\mathrm{Fe} / \mathrm{H}]$ have comparable values of 0.26 dex when we include only the RGB phase and of 0.27 dex when we include only the AGB phase. In addition, if we randomly assign $22 \%$ of the stars within the RGB box with metallicities as derived using only the AGB phase, while the remaining $78 \%$ of the stars with metallicities as derived using only the RGB phase, then the resulting MDF has a mean of $\langle[\mathrm{Fe} / \mathrm{H}]\rangle=-1.24 \mathrm{dex}$ with $1 \sigma$ spread in $[\mathrm{Fe} / \mathrm{H}]$ of 0.29 dex. This mean metallicity is comparable to the one we compute when we use only the RGB phase to derive the metallicities. The shape of the MDFs in all these cases does not change significantly. Thus, we can safely conclude that the presence of these contaminating old AGB stars within the RGB box does not affect the derived MDFs' properties significantly.

In Fig. 2 we overplot the metallicity distribution convolved with the errors in metallicity (solid line). Also shown in Fig. 2 (dashed lines) are fits of Gaussian distributions with the observed mean and dispersion. For each dSph we compute the mean metallicity, $\langle[\mathrm{Fe} / \mathrm{H}]\rangle$, as well as the error-weighted mean metallicity, $\langle[\mathrm{Fe} / \mathrm{H}]\rangle_{\mathrm{w}}$, along with the corresponding intrinsic $1 \sigma$ dispersions. We show them in Table 3, Cols. (2) and (3), while the error bars in Fig. 2 indicate the $1 \sigma$ dispersion of the error-weighted mean metallicities. The errors in metallicity are computed from a set of Monte Carlo simulations, in which each star is varied by its photometric uncertainties (both in color and magnitude, as given by the Dolphot output) and re-fit using the identical isochrone interpolation as described above. The $1 \sigma$ scatter of the output random realizations was then adopted as the metallicity error for each star. In the right panel of Fig. 4 we show the errors in metallicity computed as described above versus the metallicities derived for all the stars within the RGB box, here for KDG 64 as an example. The error in metallicity increases towards the metal-poor part, which is due to the spacing between the isochrones that becomes narrower towards the metal-poor part.

In order to further quantify the effect of the assumption of the constant age on the MDFs, we apply again the same analysis using two different constant ages for the isochrones in the
Table 3. Derived properties.

\begin{tabular}{lcccc}
\hline \hline Galaxy & $\begin{array}{c}\langle[\mathrm{Fe} / \mathrm{H}]\rangle \pm \sigma \\
(\mathrm{dex})\end{array}$ & $\begin{array}{c}\langle[\mathrm{Fe} / \mathrm{H}]\rangle_{\mathrm{w}} \pm \sigma \\
(\mathrm{dex})\end{array}$ & $\begin{array}{c}K-S^{a} \\
(\%)\end{array}$ & $f_{\mathrm{AGB}}$ \\
$(1)$ & $(2)$ & $(3)$ & $(4)$ & $(5)$ \\
\hline KDG 61 & $-1.65 \pm 0.28$ & $-1.49 \pm 0.26$ & 16 & 0.07 \\
KDG 64 & $-1.72 \pm 0.30$ & $-1.57 \pm 0.23$ & 12 & 0.09 \\
DDO 71 & $-1.64 \pm 0.29$ & $-1.56 \pm 0.24$ & 0 & 0.09 \\
F12D1 & $-1.56 \pm 0.27$ & $-1.43 \pm 0.34$ & 8 & 0.07 \\
DDO 78 & $-1.51 \pm 0.35$ & $-1.36 \pm 0.20$ & 0 & 0.09 \\
DDO 44 & $-1.77 \pm 0.29$ & $-1.67 \pm 0.19$ & 0 & 0.11 \\
IKN & $-1.38 \pm 0.37$ & $-1.08 \pm 0.16$ & $\ldots$ & 0.08 \\
F6D1 & $-1.63 \pm 0.30$ & $-1.48 \pm 0.43$ & 0.036 & 0.03 \\
HS 117 & $-1.65 \pm 0.32$ & $-1.41 \pm 0.20$ & 55 & 0.14 \\
\hline
\end{tabular}

Notes. ${ }^{(a)}$ The probabilities indicate whether the populations under consideration are from the same distribution.

interpolation method. The first constant age for the isochrones is $10.5 \mathrm{Gyr}$, while the second constant age is $8.5 \mathrm{Gyr}$. We repeat the isochrone interpolation with the bounding boxes and the metallicity ranges being kept the same in all cases. We show the results for the MDFs of all the dSphs in Fig. 5, where the MDFs for the 10.5 Gyr isochrones are shown in the left panels and the ones for the $8.5 \mathrm{Gyr}$ isochrones in the middle panels. The derived error-weighted mean metallicities $\langle[\mathrm{Fe} / \mathrm{H}]\rangle_{w, 10.5}$ and $\langle[\mathrm{Fe} / \mathrm{H}]\rangle_{w, 8.5}$, for the $10.5 \mathrm{Gyr}$ and $8.5 \mathrm{Gyr}$ isochrones, respectively, are shown in Table 4, along with their corresponding dispersions.

In addition, the star-by-star difference in $[\mathrm{Fe} / \mathrm{H}]$ as derived using the 10.5 Gyr and 8.5 Gyr isochrones is shown in Fig. 5, right panels. The maximum difference in the derived $[\mathrm{Fe} / \mathrm{H}]$ using the 10.5 Gyr isochrones minus the isochrones with a constant age of $12.5 \mathrm{Gyr}$ is less than $0.20 \mathrm{dex}$ in all the cases, while the maximum difference in the derived $[\mathrm{Fe} / \mathrm{H}]$ using the $8.5 \mathrm{Gyr}$ isochrones minus the isochrones with a constant age of $12.5 \mathrm{Gyr}$ is less than 0.40 dex in all the cases. Finally, the overall shape of the MDFs as derived for the 10.5 Gyr and the 8.5 Gyr isochrones does not change significantly.

\subsection{Population gradients}

In order to examine the presence or absence of population gradients in our dSph sample, we construct the cumulative histograms of the stars in each dSph selected in two metallicity ranges, defined as above and below the respective $\langle[\mathrm{Fe} / \mathrm{H}]\rangle_{\mathrm{w}}$. Since the dSphs can be considered as being elliptical in projection to first order, we define in the following the elliptical radius $r$ as

$$
r=\sqrt{x^{2}+\frac{y^{2}}{(1-\epsilon)^{2}}},
$$

where $x$ and $y$ are the distance along the major and minor axis, and $\epsilon$ is the ellipticity. The major and minor axes are computed by fitting an ellipse to contours of the number counts of all stars above the $1 \sigma$ level. This ellipse is shown in white in Fig. 6 and represents the elliptical shape that was chosen for each dSph. In the same Fig. 6 we show the contours above the $0.5 \sigma$ to $2 \sigma$ level, which are overlaid on top of density maps. In the study of population gradients we exclude the IKN dSph since the field of view does not cover the whole extent of the galaxy and furthermore is contaminated by a bright foreground star. In addition, we do not show the elliptical shape for IKN. 

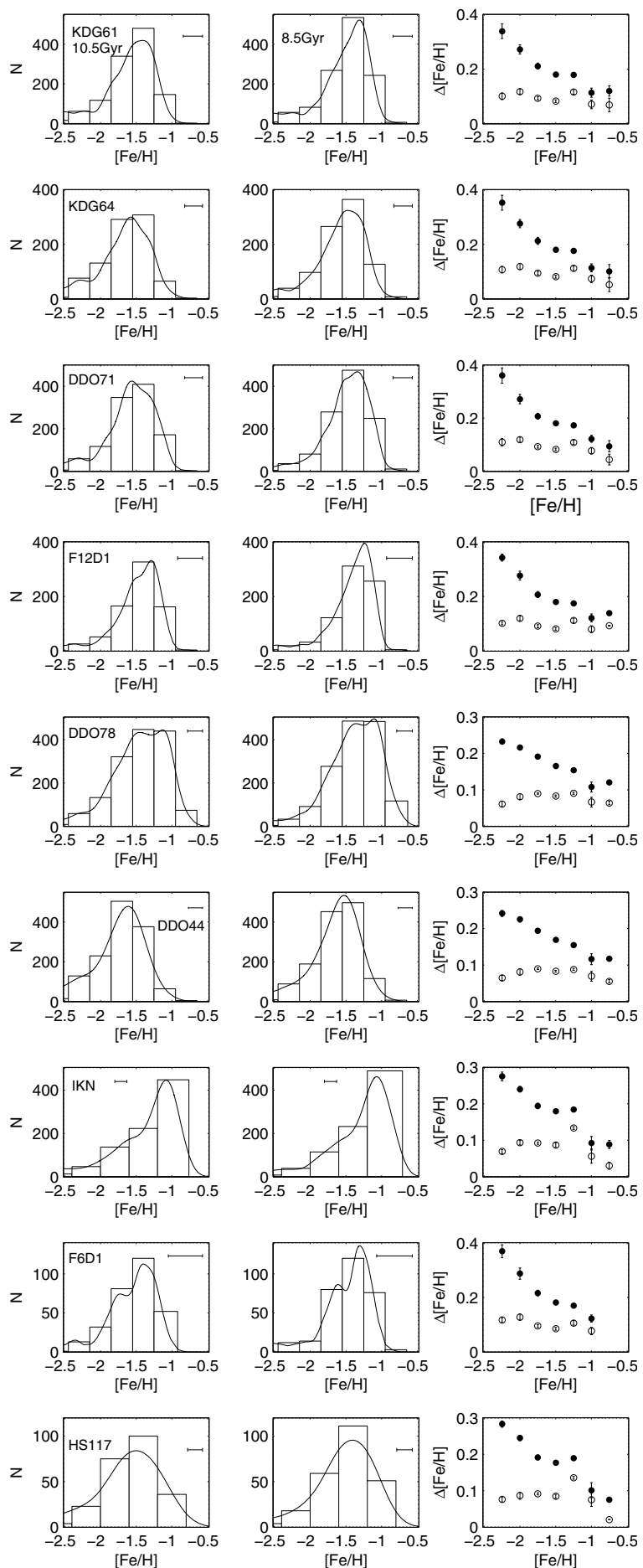

Fig. 5. Metallicity distribution functions for the nine dSphs computed in the same way using isochrones with two different ages: left panels for a constant age of $10.5 \mathrm{Gyr}$ and middle panels for a constant age of 8.5 Gyr. The solid lines show the metallicity distribution convolved with the errors in metallicity. The error bars correspond to the $1 \sigma$ spread for the weighted mean metallicity we derive from our data. Right panels: star-by-star difference of the derived metallicities, $\Delta[\mathrm{Fe} / \mathrm{H}]$, using the $10.5 \mathrm{Gyr}$ isochrones minus the $12.5 \mathrm{Gyr}$ isochrones versus the $[\mathrm{Fe} / \mathrm{H}]$ of the $10.5 \mathrm{Gyr}$ isochrones, indicated with the open circles. The star-by-star differences of the $8.5 \mathrm{Gyr}$ isochrones minus the $12.5 \mathrm{Gyr}$ isochrones are indicated with the dots.

We show the cumulative metallicity distributions in Figs. 7 and 8 (middle panels). We show in the same figures the radial metallicity distributions (upper panels) and the mean radial
Table 4. Error-weighted mean metallicities for the 10.5 Gyr and 8.5 Gyr isochrones MDFs.

\begin{tabular}{lcc}
\hline \hline Galaxy & $\begin{array}{c}\langle[\mathrm{Fe} / \mathrm{H}]\rangle_{\mathrm{w}, 10.5} \pm \sigma \\
(\mathrm{dex})\end{array}$ & $\begin{array}{c}\langle[\mathrm{Fe} / \mathrm{H}]\rangle_{\mathrm{w}, 8.5} \pm \sigma \\
(\mathrm{dex})\end{array}$ \\
$(1)$ & $(2)$ & $(3)$ \\
\hline KDG 61 & $-1.37 \pm 0.27$ & $-1.32 \pm 0.27$ \\
KDG 64 & $-1.45 \pm 0.25$ & $-1.39 \pm 0.26$ \\
DDO 71 & $-1.41 \pm 0.25$ & $-1.34 \pm 0.27$ \\
\hline F12D1 & $-1.31 \pm 0.35$ & $-1.25 \pm 0.36$ \\
DDO 78 & $-1.28 \pm 0.21$ & $-1.24 \pm 0.22$ \\
DDO 44 & $-1.60 \pm 0.20$ & $-1.53 \pm 0.20$ \\
IKN & $-1.03 \pm 0.17$ & $-0.98 \pm 0.17$ \\
F6D1 & $-1.37 \pm 0.48$ & $-1.31 \pm 0.50$ \\
HS 117 & $-1.31 \pm 0.21$ & $-1.27 \pm 0.22$ \\
\hline
\end{tabular}

metallicity profiles (lower panels). Each radial metallicity profile shows the mean values of metallicity within an elliptical annulus versus the elliptical annulus in units of the effective radius $r_{\mathrm{eff}}$. The values for $r_{\mathrm{eff}}$ are listed in the Col. (10) of Table 2. The error bars in the metallicity profile correspond to the standard deviation of the mean metallicity in each elliptical radius annulus.

\subsection{Density maps}

We now examine the spatial distribution of the stellar populations, separated into a metal-poor and metal-rich component. For that purpose, we define two stellar populations, the first includes stars having a metallicity less than or equal to the value of -1.80 dex ("metal-poor"), while the second includes stars with a metallicity larger than or equal to -1.30 dex ("metalrich"). All galaxies have peak values that lie well in between those cuts so that the metal-poor and the metal-rich tails are representatively sampled for all dSphs. For these two populations we construct the Gaussian-smoothed density maps, shown in Figs. 9 and 10, upper and middle panels for each dSph.

\subsection{Luminous AGB stars}

As already noted before, all the dSphs in our sample contain a significant number of luminous AGB star candidates. These stars are located above the TRGB and have ages ranging from $1 \mathrm{Gyr}$ up to less than 10 Gyr. We broadly refer to stellar populations in this age range as "intermediate-age" populations. Assuming that the metallicities of dwarf galaxies increase with time as star formation continues, we may also assume that these intermediateage populations are more metal-rich than the old populations. We note, however, that dwarf galaxies do not necessarily experience smooth metal enrichment as a function of time (see, e.g., Koch et al. 2007a, 2007b).

In Figs. 9 and 10, the density maps in the lower panels show the spatial distribution of these intermediate-age stars for each $\mathrm{dSph}$. We consider as luminous AGB stars the stars that are brighter by 0.15 mag than the TRGB (Armandroff et al. 1993) and that lie within 1 mag above $\left(I_{\text {TRGB }}-0.15\right)$ mag. In addition, we consider stars within the color range of $a<(V-I)_{0}<$ $a+2.50$ (mag), where the left-hand limit $a$ is equal to the color of the TRGB of the most metal-poor isochrone we use, dereddened for each $\mathrm{dSph}$ using the extinction values listed in Cols. (6) and (7) of Table 2. Then, the right-hand limit is the left-hand limit plus $2.50 \mathrm{mag}$. This selection criterion was motivated by the work of Brewer et al. (1995) and Reid \& Mould (1984). 

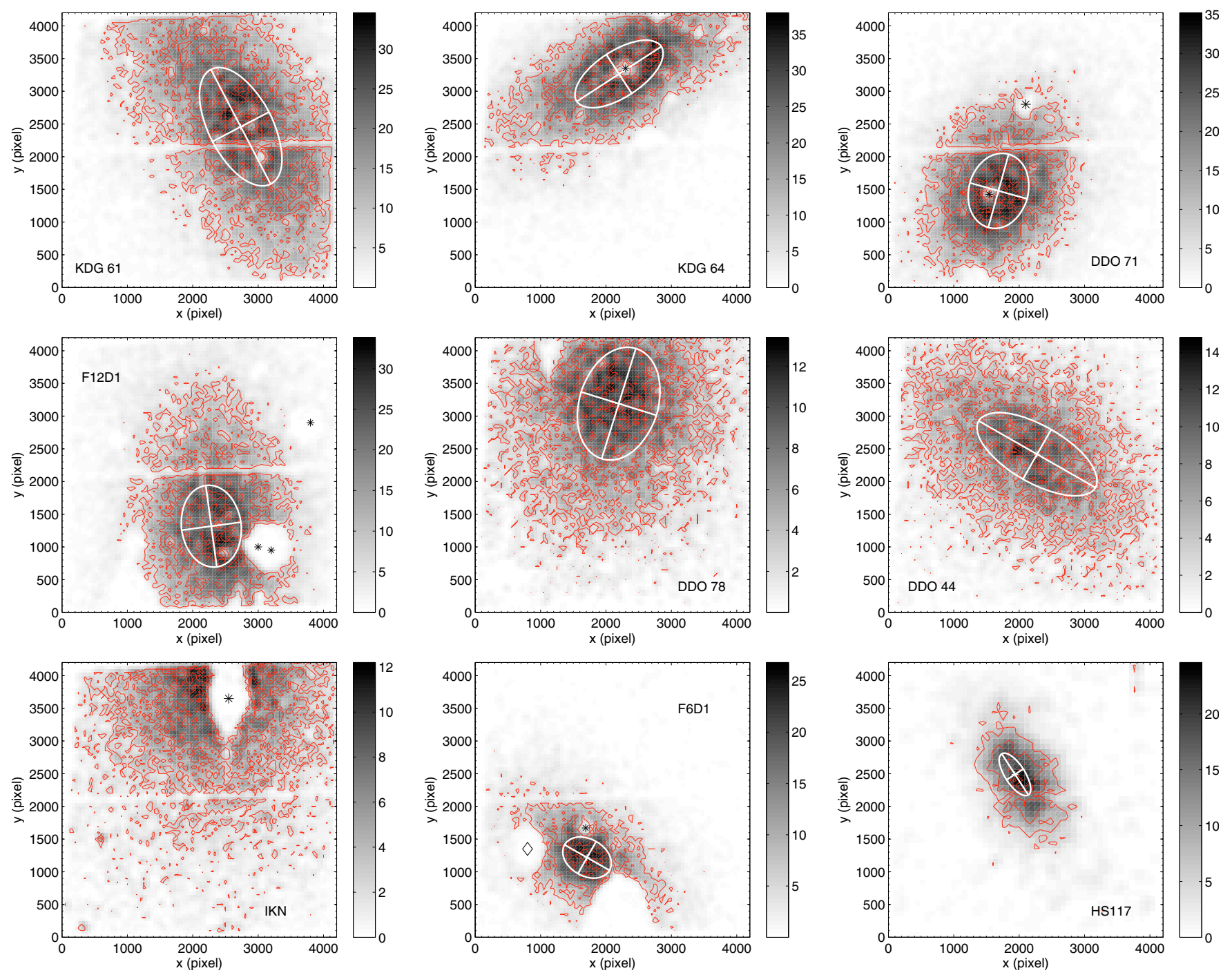

Fig. 6. Contour plots, shown in red, for the nine dSphs which are overlaid on top of density maps. The elliptical shape chosen for each dSph, with the exception of IKN, is shown with the white ellipse. The star symbols in some plots correspond to bright foreground stars, while in the case of KDG 64 corresponds to a background galaxy. The diamond symbol in the case of F6D1 corresponds to an extended background galaxy. The unit of the colorbars is number of stars per (50 pixels) $)^{2}$.

\section{Discussion}

\subsection{Photometric metallicity distribution functions}

The photometric MDFs in Fig. 2 indicate that these dSphs cover a wide range in metallicity. All of them seem to have a steeper cut-off in their metal-rich end. This can be easily seen if we compare the MDFs to the fitted Gaussian distributions, indicated by the dashed lines in Fig. 2. We do not expect the MDFs to follow a Gaussian distribution since they are shaped by the star formation histories of each $\mathrm{dSph}$. For instance, a steep cut-off of the MDF toward the metal-rich tail could be indicative of the occurrence of strong and continuous galactic winds (Lanfranchi \& Matteucci 2003, 2007; Koch et al. 2006) or of the effects of extended star formation and SNe Ia "pockets" of localized, inhomogeneous enrichment (Marcolini et al. 2008).

The low mean metallicities, $\langle[\mathrm{Fe} / \mathrm{H}]\rangle$, that are derived from the distribution functions and are shown in Table 3, Cols. (2) and (3), indicate that the M $81 \mathrm{dSphs}$ are metal-poor systems, which points to a low star formation efficiency in analogy with the LG dSphs (e.g., Lanfranchi \& Matteucci 2003; Grebel et al. 2003; Koch 2009).
One exception is the dSph IKN, which shows a high mean metallicity for its luminosity. Objects that have high metallicity for their luminosity and that, most importantly, are dark matter free are promising candidates for tidal dwarf galaxies. Their properties are set by their formation mechanism. They are believed to form out of the dark matter free material that was expelled during the tidal interaction of the parent galaxies (Bournaud et al. 2007; and references therein). One possible tidal dwarf galaxy candidate has been identified in the M 81 group, namely Holmberg IX (Makarova et al. 2002; Sabbi et al. 2008) and these are favoured to be detected in such recently interacting groups. These systems contain a young stellar component, while their older stellar populations are believed to consist of stars from their parent galaxies, which is M 81 in the case of Holmberg IX. There is no information available about the presence or absence of dark matter in this system.

In the case of IKN, we should consider the fact that its stellar metallicity bears the imprint of the medium that formed these old stars, while young stars are not observed in this dwarf. That makes it distinct from young tidal dwarf candidates like Holmberg IX. A connection with the recent interactions of the 
S. Lianou et al.: M 81 group dSphs - MDFs and population gradients
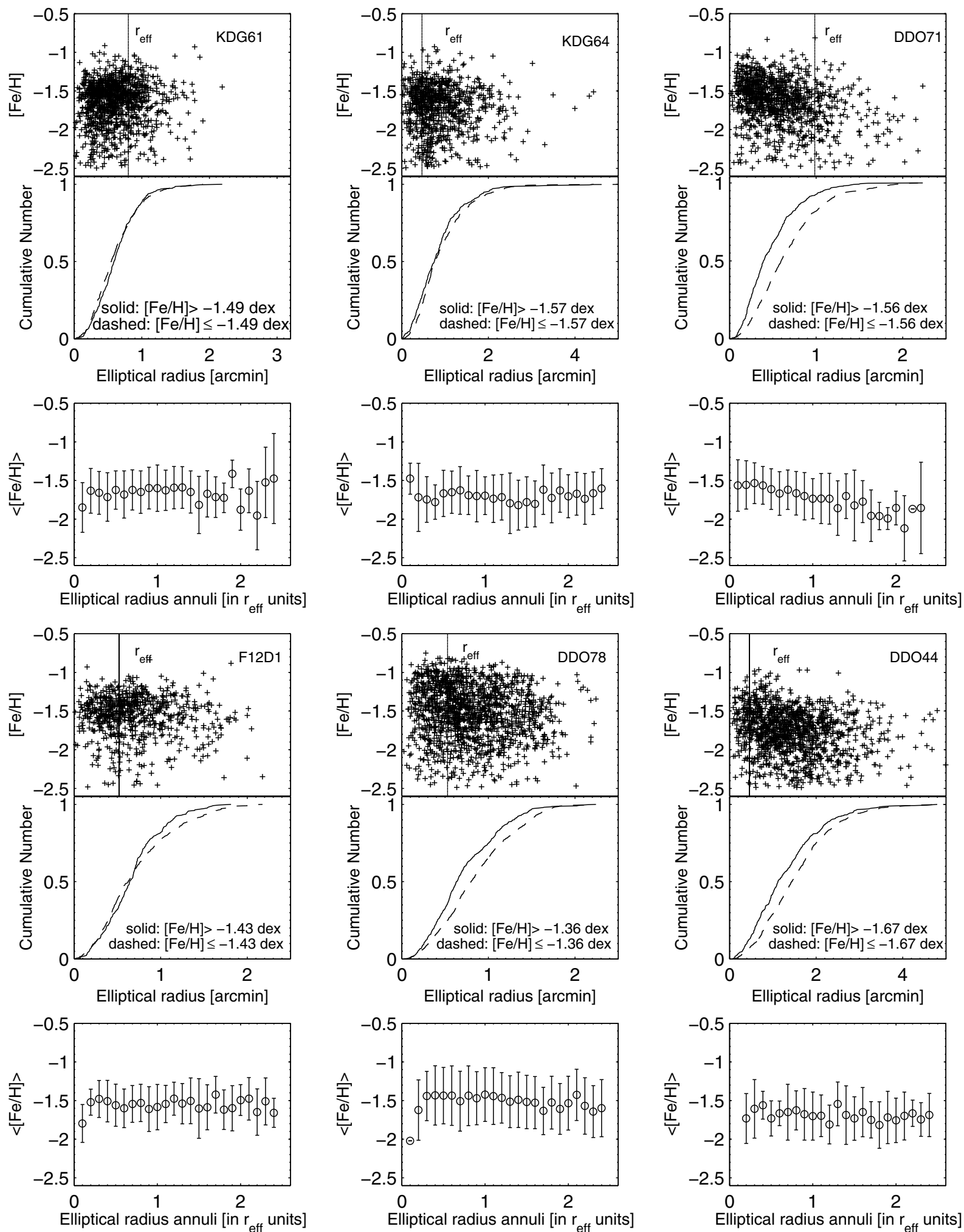

Fig. 7. In each panel from left to right and from top to bottom we show the radial metallicity distributions (top panels), their cumulative distributions (middle panels), and the radial mean metallicity profile (bottom panels). The cumulative histogram is for stars selected in metallicity above and below the weighted mean value $\langle[\mathrm{Fe} / \mathrm{H}]\rangle_{\mathrm{w}}$ listed in Table 3 .

M 81 group is not obvious. IKN might be an old tidal dwarf galaxy if such systems exist. Alternatively, IKN may have undergone substantial mass loss in the past, leaving it as a lowluminosity but comparatively high-metallicity dSph. Without data on its detailed structure and kinematics, we cannot distinguish between these possibilities.
The metallicity spreads of the studied dSphs are large, spanning $1 \sigma$ ranges from 0.27 dex to $0.37 \mathrm{dex}$, or intrinsic, errorweighted $1 \sigma$ ranges from 0.16 dex to 0.43 dex. These abundance spreads are comparable to the ones observed in the LG dSphs (Grebel et al. 2003; Koch 2009) and may indicate the presence of multiple stellar populations and/or extended star formation 

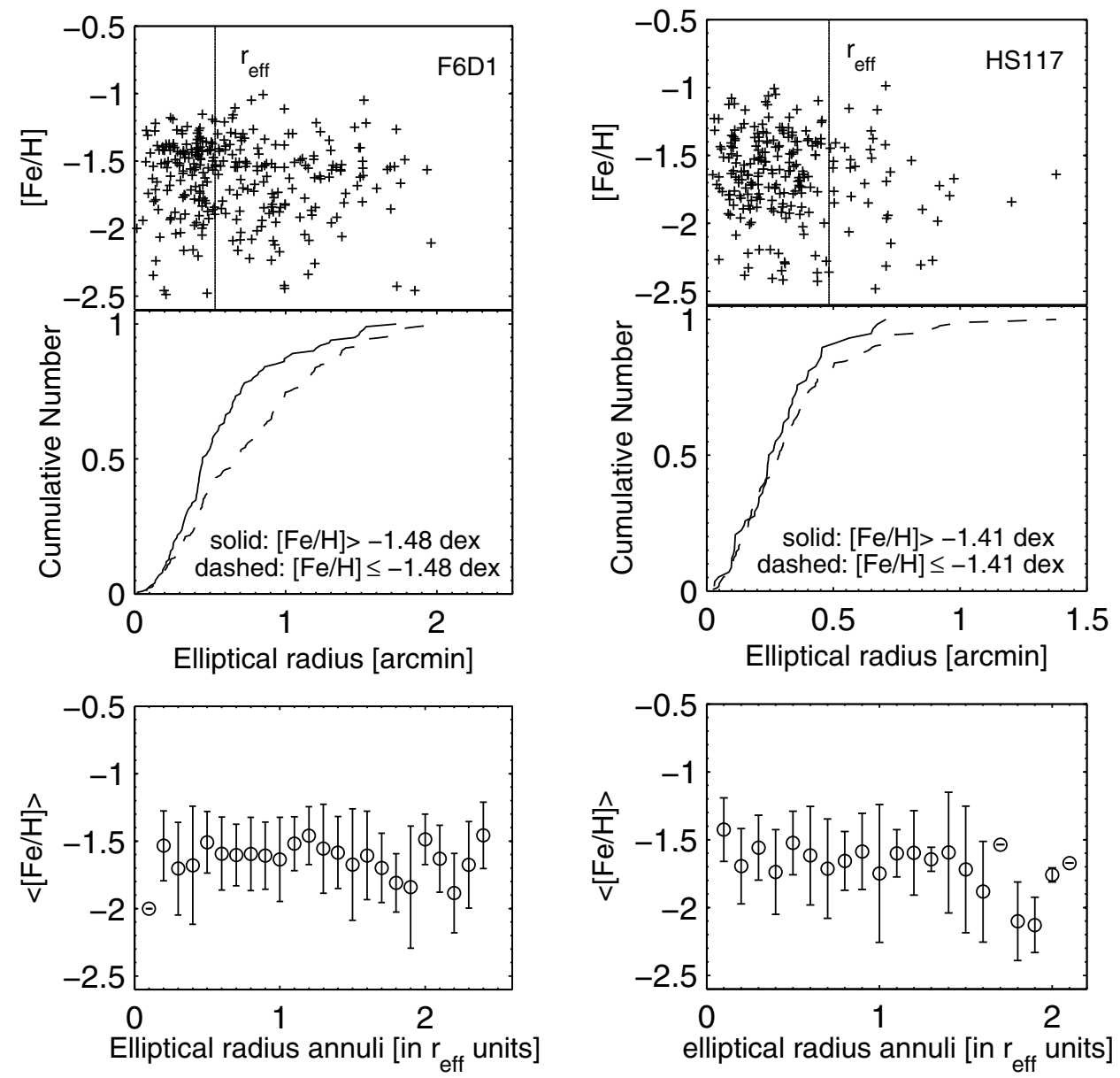

Fig. 8. Same as in Fig. 7 for the remaining two dSphs. Note that IKN is excluded from this analysis.

histories. According to the models of Marcolini et al. (2008) and Ikuta \& Arimoto (2002), the initial star formation in dSphs may have lasted as long as $3 \mathrm{Gyr}$ or even longer, which can lead to a large dispersion in $[\mathrm{Fe} / \mathrm{H}]$. For ages older than $10 \mathrm{Gyr}$, the shape of the MDF does not depend strongly on age as described in Sect. 3.2 and shown in Fig. 5.

\subsection{Luminosity-metallicity relation}

In Fig. 11, upper panel, we show the luminosity-metallicity relation compiled for the dwarf galaxies in our LG as studied by Grebel et al. (2003), with the addition of thirteen dSphs in the M 81 group. This compilation includes LG objects with mean metallicities derived from either spectroscopic or photometric studies. The mean metallicities for the nine M 81 group dSphs listed in Table 3, Col. (3), are from this work, while the metallicities for the remaining four $\mathrm{dSphs}$ are adopted from the literature and are computed using the mean $(V-I)_{0}$ color of the RGB stars at the luminosity of $M_{I}=-3.5 \mathrm{mag}$ (from Caldwell et al. (1998) for BK5N and F8D1 and from Sharina et al. (2008) for KKH 57 and $\mathrm{BK} 6 \mathrm{~N})$.

Overall, the M81 group dwarfs follow the luminositymetallicity relation quite well, albeit some of them exhibit a tendency of being slightly more metal-poor than LG dSphs of comparable luminosity. Therefore, they mainly populate the region defined by the LG dSphs while a few are located in the border region between the dSph and dIrr locus defined by the LG dwarfs. The M 81 group dSphs that seem to lie in this apparent transition region are KDG 61, KDG 64, DDO 44 and DDO 71. Out of these four objects, three are classified as transition-types, namely, KDG 61, KDG 64 and DDO 71 (Karachentsev \& Kaisin 2007; Boyce et al. 2001) based on $\mathrm{HI}$ detections and $\mathrm{H} \alpha$ emission. Also among the dwarfs that coincide with the LG dSph locus, one dwarf is classified as transition-type, namely HS 117, with HI associated with it (Huchtmeier \& Skillman 1998; Karachentsev et al. 2006).

Transition-type dwarfs are galaxies that share properties of both morphological types. Their stellar populations and star formation histories resemble those of dSphs and their gas content and present-day star formation activity is akin to low-mass dIrrs. It has been suggested that transition-type dwarfs are indeed evolving from dIrrs to gas-deficient dSphs.

The projected spatial distribution of the dSphs within the M 81 group is shown in Fig. 1 of Karachentsev et al. (2002), while their three-dimensional view is shown in their Fig. 6. In Fig. 11, middle panel, we plot the deprojected distances from the M 81 galaxy, $R$, versus the mean metallicities for the 13 M 81 group dSphs. The deprojected distances from the M 81 galaxy, $R$, are adopted from Karachentsev et al. (2002) and are listed in Table 2, Col. (9). The most distant dSph is DDO 44 which belongs to the NGC 2403 subgroup, while KDG 61 is the one closest to the M81 itself. Interestingely, the dwarf KDG 61 which is classified as a transition-type based on HI detections, is the closest to the M 81 itself, with a deprojected distance of $44 \mathrm{kpc}$ (Karachentsev et al. 2002). The remaining three dwarfs classified as transition-types, namely KDG 64, DDO 71 and HS 117, lie in a deprojected distance of more than $100 \mathrm{kpc}$. As discussed already, the most metal-rich dSph studied by us is IKN, while 
S. Lianou et al.: M 81 group dSphs - MDFs and population gradients
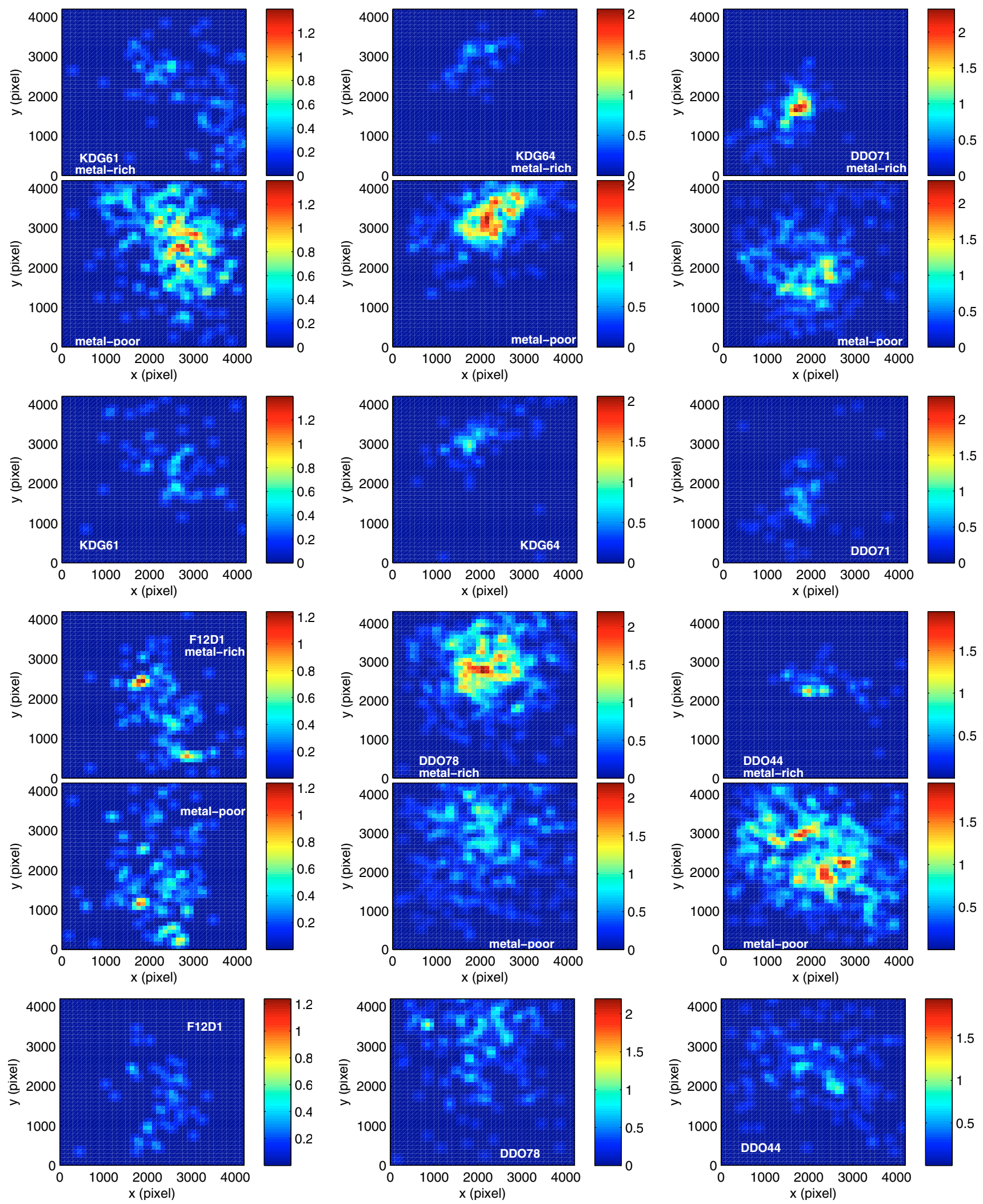

Fig. 9. From left to right and from top to bottom we show the density maps for each dSph, after smoothing with a Gaussian kernel. In each upper and middle panel, the "metal-rich" population corresponds to stars having $[\mathrm{Fe} / \mathrm{H}] \geq-1.30$ dex while "metal-poor" refers to $[\mathrm{Fe} / \mathrm{H}] \leq-1.80$. The bottom panel corersponds to the density map of the luminous AGB stars, as defined in the text. The unit of the colorbars is number of stars per $(100 \text { pixels })^{2}$. 

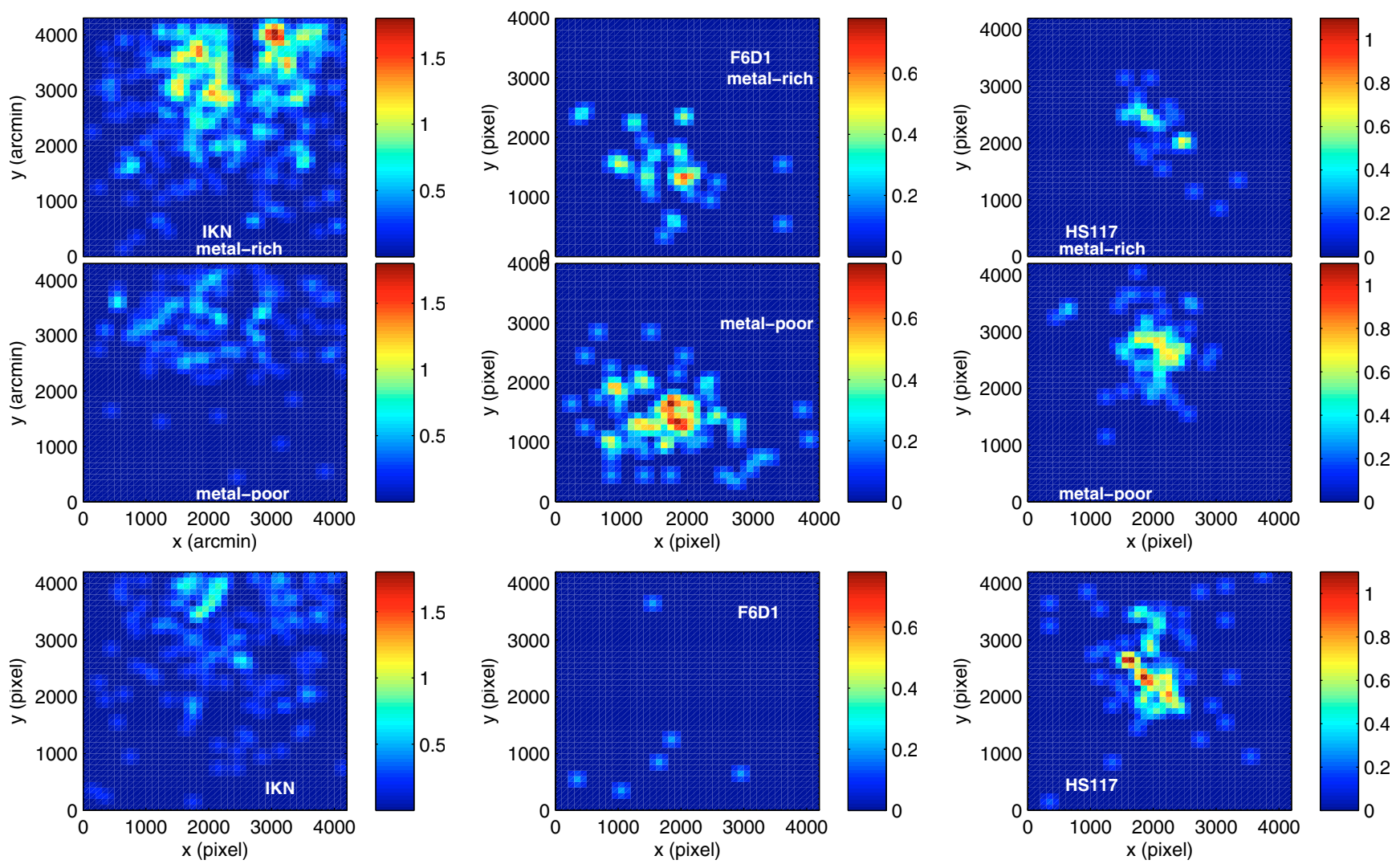

Fig. 10. The same as in Fig. 9 but for the remaining three dSphs. Note that the zero density region in the center of IKN is an artifact due to a bright star contaminating the field of view.

according to the value that Caldwell et al. (1998) provide for F8D1, this is the most metal-rich dSph in this group so far studied. We see no trend of the mean metallicity with the deprojected distance.

\subsection{Population gradients}

By examining the cumulative metallicity distributions in the middle panels in Figs. 7 and 8, we conclude that the metallicity gradients are present in the case of DDO 71, DDO 78, DDO 44 and F6D1, while the metallicity gradients are less pronounced or not present in the remaining dSphs. We again separate the RGB stars in each dSph into two samples, where we choose to separate the distributions at the observed weighted mean metallicity. The probabilities from the two-sided Kolmogorov-Smirnov $(\mathrm{K}-\mathrm{S})$ test that the two components are drawn from the same parent distribution are listed in Table 3, Col. (4). The K-S results are consistent with showing spatially separated populations in the case of DDO 71, DDO 78, DDO 44 and F6D1. In the case of the remaining $\mathrm{dSphs}$, the gradients are less pronounced and the metal-rich and metal-poor populations have different distributions at the $84-99.7 \%$ confidence level $(>1.5 \sigma)$, except for HS 117. In all cases, in which we observe such metallicity segregation, the sense is that more metal rich stars are more centrally concentrated, as also found in the majority of the LG dSphs (Harbeck et al. 2001; Tolstoy et al. 2004; Ibata et al. 2006).

\subsection{Density maps}

The density maps in Figs. 9 and 10 are useful to study the spatial distribution of the metal-rich (upper panels) and metalpoor (middle panels) population of each dSph. From these density maps we conclude that each dSph has a different stellar spatial distribution of their metal-rich and metal-poor stellar component. All of them show either a spatial variation of the centroids of the two stellar populations, as is the case of F12D1 and KDG 64, or that the metal-rich population is more centrally concentrated, as is the case of DDO 71, DDO 44 and F6D1. These findings agree well with the ones from the cumulative histograms, though we should keep in mind that the metalpoor and metal-rich stellar populations involved are differently selected. DDO 78 and IKN are clearly fairly metal-rich while KDG 61, KDG 64 and DDO 44 have prominent metal-poor populations.

\subsection{Luminous AGB stars}

Luminous AGB stars were also detected in two more dSphs in the M 81 group, namely BK5N and F8D1 (Caldwell et al. 1998). These luminous AGB stars may include carbon stars and may be long-period variables (LPVs) as have been found in other early-type dwarf galaxies (e.g., Menzies et al. 2002; Rejkuba et al. 2006; Whitelock et al. 2009), but we can not establish this for certain based on our current data. We compute the fraction of the luminous AGB stars, $f_{\mathrm{AGB}}$, defined as the number of the luminous AGB stars, $N_{\mathrm{AGB}}$, counted within the magnitude bin considered in Sect. 3.5, over the number of the RGB stars within one magnitude below the TRGB, $N_{\mathrm{RGB}}$. In order to estimate the $N_{\mathrm{RGB}}$, we take into account that approximately $22 \%$ of the stars we count within one magnitude below the TRGB are old AGB stars (Durrell et al. 2001). Thus, the $N_{\text {RGB }}$ is equal to $78 \%$ of the stars we count within one mag below the TRGB. The fractions $f_{\mathrm{AGB}}$ we derive in this way are listed in Table 3, Col. (5). 


\subsubsection{Blends, blue straggler progeny, and foreground contamination}

We now discuss the possibility that these luminous AGB stars may actually be (1) blends of bright RGB stars (Renzini 1998), (2) blue straggler progeny (Guarnieri et al. 1997; and references therein), or (3) due to the foreground contamination.

In order to evaluate case (1), we use our artificial star experiments in order to quantify the number of the blends of bright RGB stars, $N_{\text {blends, }}$, that may contribute to the detected number of the observed luminous AGB stars. We only consider here the case of a blend of two equal-magnitude stars. The magnitude of the blended star is always 0.75 mag brighter than the initial magnitude of the two superimposed stars.

We want to determine the location in the CMD of all the RGB stars that can end up as blends within the location in the CMD of the luminous AGB stars, as defined in Sect. 3.5 and further called as luminous AGB box. For that purpose, if we assume that the stars within the luminous AGB box were all blends, then they would originate from stars that have magnitudes $0.75 \mathrm{mag}$ fainter. Thus, we shift the luminous AGB box by 0.75 mag towards the fainter magnitudes and furthermore we only consider the stars with magnitudes fainter than the TRGB. This procedure defines the location of the RGB stars that can end up as blends within the luminous AGB box and we call this the "RGB blends box".

From the stellar catalogue we use as an input for the artificial star experiments, we select the stars that have such input magnitudes to place them within the "RGB blends box". From these input stars, we consider as blends the ones that have output magnitudes that can place them above the TRGB. We normalize the number of these blends to the number of the total input stars that are located within the "RGB blends box". Finally, the number of the observed blends is proportional to the number of the observed RGB stars located within the same "RGB blends box". Thus, the $N_{\text {blends }}$ for all the dSphs computed this way is equal to 5 blends, 11 blends, 12 blends and 2 blends in the case of DDO 44, DDO 78, IKN and HS 117, respectively, while in all the other cases the number of blends is less than 1 . Thus, the fraction of the blends defined as the number of blends divided by the number of the RGB stars within 1 mag below the TRGB, is less than $0.6 \%$ in all cases but for IKN which is equal to $0.9 \%$.

In the case (2), Guarnieri et al. (1997) point out that the number of blue straggler progeny is of the order of $\sim 2 \%$ of all stars that reach the luminous AGB phase.

In the case (3), we estimate the foreground contamination using the TRILEGAL code (Vanhollebeke et al. 2009; Girardi et al. 2005). We count the number of foreground stars that fall within the same location in the CMD as in the luminous AGB box and these are considered to be the number of expected foreground contamination. In all the cases, the number of foreground stars is 4, with the exception of DDO 71 and DDO 44 where the number of foreground stars is 3 and 5, respectively. This translates to a fraction of foreground stars, defined as the number of foreground stars divided by the number of RGB stars within 1 mag below the TRGB, of less than $0.7 \%$, with the exception of F6D1 where this fraction is equal to $2 \%$.

We do not consider the case of old AGB LPVs, whose large amplitude variations may place them above the TRGB (Caldwell et al. 1998), as an additional source of contamination in the luminous AGB box, since the studied dSphs have mean metallicities of less than -1 dex. Such old AGB LPVs, with ages greater than or equal to $10 \mathrm{Gyr}$, have been observed above the TRGB in metal-rich $([\mathrm{Fe} / \mathrm{H}]>-1$ dex $)$ globular clusters (e.g., Guarnieri et al. 1997; and references therein).

We can now add all the contributions estimated in the above three cases, for each dSph. We call the sum of these three contributions the number of total contaminants, $N_{\text {cont,tot }}$, and compute their fraction $f_{\text {cont,tot }}=N_{\text {cont,tot }} / N_{\text {RGB }}$. The fraction of the total contaminants is less than $1 \%$ in all cases apart from IKN, HS 117 and F6D1, where the fraction of the total contaminants is approximately $1.1 \%, 1.3 \%$ and $2.1 \%$, respectively. We note that in all cases, there is a significant fraction of luminous AGB stars that can not be accounted for by considering the contribution of blends, binaries and foreground contamination. The dSph F6D1 is an exception to that, where the $f_{\text {cont,tot }}$ is $\sim 2 \%$, as compared to the $f_{\mathrm{AGB}}$ which is $\sim 3 \%$. We note though that in the case of F6D1 the number of stars counted in the luminous AGB box is equal to 6 stars. Thus, we conclude that the luminous AGB stars are a genuine population for the majority of the dSphs studied here.

\subsubsection{Luminous $A G B$ density maps and fractions}

From the density maps of the luminous AGB stars shown in Figs. 9 and 10, lower panels, we see that if we consider the peak densities or the bulk of the luminous AGB stars, then it seems that these are more confined to the central regions of the dwarfs, a behaviour similar to what is found for the Fornax dSph (Stetson et al. 1998). If we consider the overall distribution then we note that for most of the $\mathrm{dSphs}$ these stars are rather more widely distributed, following the distribution of the metal-poor stars, with the exception of KDG 64 and DDO 71 where their AGB stars' distributions coincide mostly with the metal-rich population, which in the case of DDO 71 is centrally concentrated. We conclude that the intermediate-age stellar component is wellmixed with the old stellar component.

This behaviour is similar to the AGB stars' spatial distribution of the LG dwarfs. Indeed, Battinelli \& Demers (2004a; and references therein) discuss that for the LG dwarfs, for which there are carbon star studies, their Carbon-rich stars are distributed such that they coincide with the spatial distribution of the old stellar component. An exception is the dE NGC 185, where the AGB stars are concentrated more in the centre than the old stellar component (Battinelli \& Demers 2004b), a similar behaviour as observed in the two M 81 group dSphs discussed above.

We plot the $f_{\mathrm{AGB}}$ versus the deprojected distance from $\mathrm{M} 81$, $R$, in the lower panel of Fig. 11. The highest fraction of luminous AGB stars is observed in HS 117 and the lowest one in KDG 61 and F12D1. We do not see any trend of the $f_{\mathrm{AGB}}$ with increasing deprojected distance from M 81. If we compute the net fraction of the luminous AGB stars, by subtracting the fraction $f_{\text {cont,tot }}$, due to the contribution of blends, binaries and foreground contamination, from the fraction $f_{\mathrm{AGB}}$ listed in the Col. (5) of Table 3, none of the trends and conclusions change.

\section{Conclusions}

We use the CMDs of nine dSphs in the M 81 group to construct their photometric MDFs. These MDFs show populations covering a wide range in metallicity with low mean metallicities indicating that these are metal-poor systems. All MDFs show a steeper fall-off at their high-metallicity end than toward their low-metallicity end indicating that galactic winds may play a role in shaping their distribution.

We compute the mean metallicity, $\langle[\mathrm{Fe} / \mathrm{H}]\rangle$, and the mean metallicity weighted by the metallicity error, $\langle[\mathrm{Fe} / \mathrm{H}]\rangle_{\mathrm{w}}$, along 

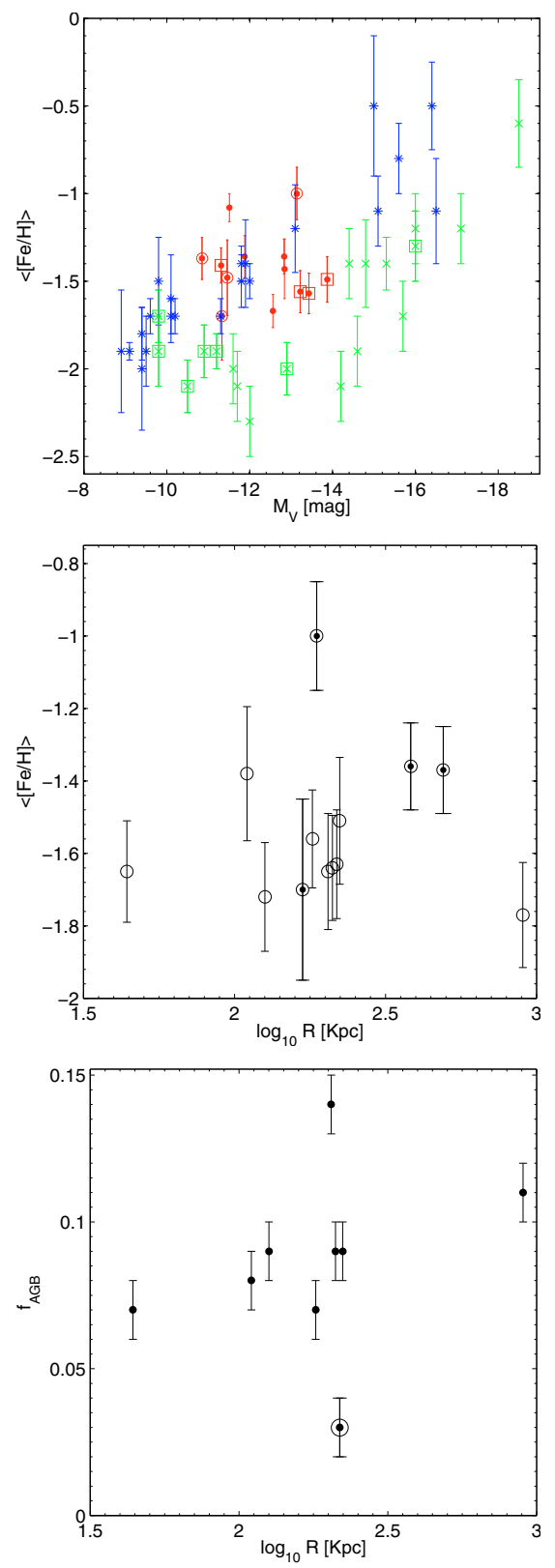

Fig. 11. Upper: luminosity-metallicity relation for LG dwarf galaxies, after Grebel et al. (2003), together with the $13 \mathrm{dSphs}$ of the M 81 group. LG dSphs are plotted with blue asterisks, LG dIrrs are shown as green crosses, and red dots indicate the available M 81 data. Nine out of the thirteen M 81 group dSphs have been studied here, while the remaining four, marked with a red circled dot, have been adopted from the literature, as discussed in the text. The red squared dots and green squared crosses indicate the transition-types of the M 81 group and LG, respectively. Middle: mean metallicities versus the deprojected distance from $\mathrm{M} 81, R$, for the $13 \mathrm{M} 81$ group dSphs. The circled dots correspond to the four dSphs for which the metallicities were adopted from the literature, as discussed in the text. Lower: fraction of AGB stars versus the RGB stars within 1 mag below the TRGB, $f_{\mathrm{AGB}}$, versus the deprojected distance from M 81, $R$, for the nine M 81 group dSphs studied here. With the circled dot we show the corresponding fraction $f_{\mathrm{AGB}}$ in the case of F6D1.

with their corresponding standard deviations. The most metalrich dSph in our sample is IKN even though it is the least luminous galaxy in our sample. IKN's comparatively high metallicity may indicate that it is a tidal dwarf galaxy or that it suffered substantial mass loss in the past. We do not see any correlation between the $\langle[\mathrm{Fe} / \mathrm{H}]\rangle$ and the deprojected distance from the M 81 galaxy, $R$.

We use the mean metallicity weighted by the metallicity errors, $\langle[\mathrm{Fe} / \mathrm{H}]\rangle_{\mathrm{w}}$, to select two stellar populations having metallicities above and below that value. For these two stellar populations we construct cumulative histograms, as a way to search for population gradients in metallicity. We find that some dSphs show strong metallicity gradients, while others do not. In dSphs with radial metallicity gradients the more metal-rich populations are more centrally concentrated.

Furthermore, we study the spatial (i.e., two-dimensional) distribution of our defined metal-rich and metal-poor stellar populations. This refined look no longer assumes radial symmetry, and we now find that in some dwarfs the metal-rich population is more centrally concentrated, while others show offsets in the centroid of the two populations. By examining the distribution of the luminous AGB stars, we conclude that, for the majority of the dSphs, these stars have mostly extended distributions, indicating that they have been well-mixed with the metal-poor stellar population. We do not find any correlation between the fraction of luminous AGB stars and the deprojected distance from the M 81 galaxy. While present-day distances may not be indicative of the dwarfs' position in the past and while their orbits are unknown, the apparent lack of a correlation between distance and evolutionary history may suggest that the evolution of the dwarfs was determined to a large extend by their internal properties and not so much by their environment.

Finally, there are some M81 dSphs that straddle the transition region between $\mathrm{LG} \mathrm{dSphs}$ and dIrrs in the metallicity-luminosity relation. We may be observing lowluminosity transition-type dwarfs moving toward the dSph locus. Interestingly, these dwarfs are slightly more luminous than the bulk of the LG transition dwarfs. Perhaps some of the M 81 dwarfs experienced gas stripping during the recent interactions between the dominant galaxies in the M 81 group.

Acknowledgements. The authors would like to thank an anonymous referee for the thoughtful comments. We would like to thank Rainer Spurzem and Thorsten Lisker for useful discussions. S.L. and this research were supported within the framework of the Excellence Initiative by the German Research Foundation (DFG) via the Heidelberg Graduate School of Fundamental Physics (HGSFP) (grant number GSC 129/1). S.L. would like to acknowledge an EAS travel grant to participate to the JENAM 2008 conference in Vienna, where the preliminary results were shown. AK acknowledges support by an STFC postdoctoral fellowship and by the HGSFP of the University of Heidelberg.

This research has made use of the NASA/IPAC Extragalactic Database (NED) which is operated by the Jet Propulsion Laboratory, California Institute of Technology, under contract with the National Aeronautics and Space Administration. This research has made use of NASA's Astrophysics Data System Bibliographic Services. This research has made use of SAOImage DS9, developed by Smithsonian Astrophysical Observatory. This research has made use of Aladin.

All of the data presented in this paper were obtained from the Multimission Archive at the Space Telescope Science Institute (MAST). STScI is operated by the Association of Universities for Research in Astronomy, Inc., under NASA contract NAS5-26555. Support for MAST for non-HST data is provided by the NASA Office of Space Science via grant NNX09AF08G and by other grants and contracts.

\section{References}

Alonso-Garcia, J., Mateo, M., \& Aparicio, A. 2006, PASP, 118,580 Armandroff, T. E., Da Costa, G. S., Caldwell, N., \& Seitzer, P. 1993, AJ, 106, 986

Appleton, P. N., Davies, R. D., \& Stephenson, R. J. 1981, MNRAS, 195, 327

Battaglia, G., Tolstoy, E., Helmi, A., et al. 2006, A\&A, 459, 423

Battinelli, P., \& Demers, S. 2004a, A\&A, 418, 33

Battinelli, P., \& Demers, S. 2004b, A\&A, 417, 479

Bournaud, F., Duc, P.-A., Brinks, E., et al. 2007, Science, 316, 1166 
Boyce, P. J., Minchin, R. F, Kilborn, V. A., et al. 2001, ApJ, 560, 127 Brewer, J. P., Richer, H. B., \& Crabtree, D. R. 1995, AJ, 109, 2480 Caldwell, N., Armandroff, T. E., Da Costa, G. S., \& Seitzer, P. 1998, AJ, 115, 535

Chiboucas, K., Karachentsev, I. D., \& Tully, R. B. 2009, AJ, 137, 3009 Dolphin, A. E. 2000, PASP, 112, 1383

Dotter, A., Chaboyer, B., Jevremovic, D., et al. 2008, ApJS, 178, 89

Durrell, P. R., Harris, W. E., \& Pritchet, C. J. 2001, AJ, 121, 2557

Frayn, C. M., \& Gilmore, G. F. 2002, MNRAS, 337, 445

Georgiev, I. Y., Puzia, T. H., Hilker, M., \& Goudfrooij, P. 2009, MNRAS, 392, 879

Glatt, K., Gallagher, J. S., III, Grebel, E. K., et al. 2008a, AJ, 135, 1106

Glatt, K., Grebel, E. K., Sabbi, E., et al. 2008b, AJ, 136, 1703

Girardi, L., Groenewegen, M. A. T., Hatziminaoglou, E., \& da Costa, L. 2005, A\&A, 436, 895

Girardi, L., Dalcanton, J., Williams, B., et al. 2008, PASP, 120, 583

Grebel, E. K. 2001, Ap\&SSS, 277, 231

Grebel, E. K., \& Roberts, W. J. 1995, A\&AS, 109, 293

Grebel, E. K., \& Guhathakurta P. 1999, ApJ, 511, 101

Grebel, E. K. \& Gallagher, J. S., III 2004, ApJ, 610, 89

Grebel, E. K., Gallagher, J. S., III \& Harbeck, D. 2003, AJ, 125, 1926

Guarnieri, M. D., Renzini, A., \& Ortolani, S. 1997, ApJ, 477, L21

Harbeck, D., Grebel, E. K., Holtzman, J., et al. 2001, AJ, 122, 3092

Harris, G. L. H., Harris, W. E., \& Poole, G. B. 1999, AJ, 117, 855

Huchtmeier, W. K., \& Skillman, E. D. 1998, A\&AS, 127, 269

Hurley-Keller, D., Mateo, M., \& Grebel, E. K. 1999, ApJ, 523, 25

Ibata, R., Chapman, S., Irwin, M., Lewis, G., \& Martin, N. 2006, MNRAS, 373 , 70

Ikuta, C., \& Arimoto, N. 2002, A\&A, 391, 55

Karachentsev, I. D., \& Kaisin, S. S. 2007, AJ, 133, 1883

Karachentsev, I. D., Sharina, M. E., Grebel, E. K., et al. 1999, A\&A, 352, 399

Karachentsev, I. D., Karachentseva, V. E., Dolphin, A. E., et al. 2000, A\&A, 363, 117

Karachentsev, I. D., Sharina, M. E., Dolphin, A. E., et al. 2001 A\&A, 379, 407

Karachentsev, I. D., Dolphin, A. E., Geisler, D., et al. 2002, A\&A, 383, 125

Karachentsev, I. D., Karachentseva, V. E., Huchtmeier, W. K., \& Makarov,

D. I. 2004, AJ, 127, 2031
Karachentsev, I. D., Dolphin, A., Tully, R. B., et al. 2006, AJ, 131, 1361

Karachentseva, V. E., Karachentsev, I. D., Richter, G. M., von Berlepsch, R., \& Fritze, K. 1987, AN, 308, 247

Koch, A. 2009, AN, 330, 675

Koch, A., Grebel, E. K., Wyse, R. F. G., et al. 2006, AJ, 131, 895

Koch, A., Grebel, E. K., Kleyna, J. T., et al. 2007a, AJ, 133, 270

Koch, A., Wilkinson, M. I., Kleyna, J. T., et al. 2007b, ApJ, 657, 241

Lanfranchi, G., \& Matteucci, F. 2003, A\&A, 100, 500

Lanfranchi, G., \& Matteucci, F. 2007, A\&A, 468, 927

Lee, M. G., Freedman, W. L., \& Madore, B. F. 1993, ApJ, 417, 553

Makarova, L. N., Grebel, E. K., Karachentsev, I. D., et al. 2002, A\&A, 396, 473

Marcolini, A., Brighenti, F., \& D'Ercole, A. 2003, MNRAS, 345, 1329

Marcolini, A., D'Ercole, A., Battaglia, G., \& Gibson B. K. 2008, MNRAS, 386, 2173

Marigo, P., Girardi, L., Bressan, A., et al. 2008, A\&A, 482, 883

Martinez-Delgado, D., \& Aparicio, A. 1997, ApJ, 480, L107

Menzies, J., Feast, M., Tanabe, T., Whitelock, P., \& Nakada, Y. 2002, MNRAS, 335,923

Reid, N., \& Mould, J. 1984, ApJ, 284, 98

Rejkuba, M., da Costa, G. S., Jerjen, H., Zoccali, M., \& Binggeli, B. 2006, A\&A, 448, 983

Renzini, A. 1998, AJ, 115, 2459

Sabbi, E, Gallagher, J. S., Smith, L. J., de Mello, D. F., \& Mountain, M. 2008, ApJ, 676, L113

Sakai, S., Madore, B. F., \& Freedman, W. F. 1996, ApJ, 461, 713

Sarajedini, A., Grebel, E. K., Dolphin, A. E., et al. 2002, ApJ, 567, 915

Schlegel, D. J., Finkbeiner, D. P., \& Davis, M. 1998, ApJ, 500, 525

Sharina, M. E., Karachentsev, I. D., Dolphin, A. E., et al. 2008, MNRAS, 384, 1544

Sirianni, M., Jee, M. J., Bentez, N., et al. 2005, PASP, 117, 1049

Stetson, P. B, Hesser, J. E., \& Smecker-Hane, T. A. 1998, PASP, 110, 533

Tolstoy, E., Irwin, M. J., Helmi, A., et al. 2004, ApJ, 617, 119

Vanhollebeke, E., Groenewegen, M. A. T., \& Girardi, L. 2009, A\&A, 498, 95

Whitelock, P. A., Menzies, J. W., Feast, M. W., et al. 2009, MNRAS, 394, 795

Williams, B. F., Dalcanton, D. J., Seth, A. C., et al. 2009, AJ, 137, 419

Yun, M. S., Ho, P. T. P., \& Lo, K. Y. 1994, Nature, 372, 530 